\title{
3 Research Suare \\ Bone Resorption Induced by Titanium Implantation Through Activation of Osteoclast Formation via C3a
}

Xiaohan Liu

China Medical University

Siwen Li

China Medical University

Yuan Meng

China Medical University

Yu Fan

China Medical University

Ce Shi

Jilin Medical University

Feilong Ren

Jilin Medical University

Lin Wu

China Medical University

Jinyan Wang

China Medical University

Hongchen Sun ( $\square$ sunhongchen@cmu.edu.cn)

Department of Oral Pathology, School and Hospital of Stomatology, China Medical University, Shenyang 110002, China; https://orcid.org/0000-0001-9013-0412

\section{Research}

Keywords: Bone resorption, Complement C3a, Inflammatory reaction, Titanium implantation

Posted Date: September 28th, 2020

DOl: https://doi.org/10.21203/rs.3.rs-66781/v1

License: (1) (i) This work is licensed under a Creative Commons Attribution 4.0 International License.

Read Full License 


\section{Abstract}

Titanium implantation is widely used for dental replacement with advantages of excellent mechanical strength, corrosion resistance, chemical stability and biocompatibility. Some patients, however, are subject to the failure of implantation due to bone resorption, which closely related to the inflammatory responses without clear mechanisms. In this study, first we found that there were inflammatory responses and increases of osteoclasts in the surrounding tissues near by the titanium implant. Further, data revealed that the $\mathrm{C} 3$ was increased in the serum and surrounding tissues near by the titanium implant, and activated by classical and alternative pathways. Next, we recognized that the $\mathrm{C} 3 \mathrm{a} / \mathrm{C} 3 \mathrm{aR}$, no $\mathrm{C} 3 \mathrm{~b}$ played an important role in stimulating secretions of pro-inflammatory cytokines of TNF-a and MMP9 via transcription factors NF-kB and NFATc1. This cascade of responses to titanium implant leaded the differentiation and proliferation of osteoclasts in vivo and in vitro, bone resorption of surrounding tissues of $\mathrm{Ti}$ implant. These suggest that the cleaved C3a fragment plays predominant roles in the activation of osteoclast. Therefore, the blocking C3a activation should provide potential to prevent bone resorption and prolong the survival of biomaterial implants.

\section{Introduction}

Pure titanium ( $\mathrm{Ti}$ ) is widely used for dental implants because of its excellent mechanical strength, corrosion resistance, chemical stability and biocompatibility ${ }^{[1]}$. Some of patients, however, who have been suffered from systemic diseases, such as diabetes, dyslipidemia and osteoporosis, are prone to the failure of implantation due to bone resorption.

Although the precise mechanisms of bone resorption are not fully clarified, it is believed that the activation of osteoclasts, a bone tissue-resident innate immune cell, involves in the bone resorption leading to impair osseointegration of $\mathrm{Ti}$ implant ${ }^{[2]}$. Indeed, aberrant activation of osteoclasts contributes to the resistance of tissue to the implant by initiation of local inflammatory responses resulting in the failure of implanted biomaterials. However, the reason that initiates the local tissue inflammation after $\mathrm{Ti}$ implantation remains unclear.

Complement system, more than 30 different plasma proteins in blood and tissue fluids, plays a crucial role in the activation of inflammatory responses in response to foreign substance stimulation ${ }^{[3]}$. Complement system can be activated through three pathways ${ }^{[4]}$, the classical pathway that is triggered by antibody production, the alternative pathway that can be activated by the present of non-selfsubstance and lectin pathway that is activated by the enhanced production of lectin-type proteins that recognize and bind to carbohydrates on the surface of foreign substance ${ }^{[5]}$. The activation of each complement pathway leads to inflammation, phagocytosis and membrane attack, facilitate elimination of foreign substance ${ }^{[6]}$. The complement $\mathrm{C} 3$ serves as a convergent site for all three activation pathways and is degraded into at least two fragments, $\mathrm{C} 3 \mathrm{~b}$ and $\mathrm{C} 3 \mathrm{a}{ }^{[7]}$. The accumulation of $\mathrm{C} 3 \mathrm{~b}$ on the surface of foreign substance, can be ligated by complement receptors on phagocytic cells, such as macrophage, to 
promote clearance of foreign substance ${ }^{[8]}$. In addition, C3a recruits circulating cells, further amplifying the inflammatory process ${ }^{[9]}$. It has been shown that the enhanced gene expression of $\mathrm{C} 3$ is subjected to osteoclastogenesis in vitro ${ }^{[10]}$. C3a can mediate the release of the proinflammatory cytokines IL-6 and IL8 from osteoblasts to affect osteoclast modulation and bone resorption [11]. Blocking C3a with C3a antagonist attenuates osteoclast maturation in the bone marrow cell culture ${ }^{[11]}$. It has been reported that the expression of C3a receptor is much higher at the femur fracture site of C57BL/ 6 mouse during the entire healing period ${ }^{[12]}$. All these indicate that C3 may serve as key initiator that triggers osteoclast activation to contribute to the bone resorption and favoring failure after $\mathrm{Ti}$ implantation.

Therefore, in the present study, we evaluated the impact of C3 on osteoclastogenesis in the tissue of Ti implantation during the development of bone resorption in the mice model, and identified the activation pathway of complement system that leads to increased levels of C3. Finally, we clarified the molecular mechanisms that underlies the activation of osteoclast through $\mathrm{C} 3 \mathrm{a} / \mathrm{C} 3 \mathrm{a}$ receptor interaction.

\section{Materials And Methods}

\section{Experimental materials}

Materials used in this study were fabricated by the Institute of Metal Research, Chinese Academy of Sciences (Shenyang, China). Ti disks (diameter $33 \mathrm{~mm}$, thickness $1 \mathrm{~mm}$ ) were used for the cell culture experiments, Ti cylindrical implants (diameter $0.8 \mathrm{~mm}$, length $5 \mathrm{~mm}$ ) were used for the femoral condyle implantation. All these materials were ultrasonically cleaned, autoclaved $\left(134^{\circ} \mathrm{C} / 0.21 \mathrm{MPa}\right)$.

\section{Surgical implantation}

Ninety male C57/6L mice (10 weeks old) were used. All animals were supplied by the Experimental Animals Centre from China Medical University. The animal experiments were approved by the Animal Ethics Committee of China Medical University. Mice were randomly divided into three groups, Ti implant group $(n=30)$, defect group (without Ti implant, $n=30)$ and control group $(n=30)$. Mouse was intraperitoneally received $0.4 \mathrm{ml}$ Pentobarbital (0.3\%TIANWUDR, Tianjin, China) for whole body anesthesia. Surface of femoral condyle was shaved, and sterilized with $70 \%$ ethanol. Local anesthesia was induced using $1 \mathrm{ml}$ lidocaine (2\%TIANWUDR, Tianjin, China). The surgical site exposed with a $5 \mathrm{~mm}$ incision through the skin, fascia and periosteum, then drilled a $0.8 \mathrm{~mm}$ diameter hole into the right femoral condyle using an electric speed-regulated hand drill (Shenzhen Wincore Power ToolsMZ-06, China) under profuse irrigation with sterile saline. One Ti implant was placed on the right side, rinsed with saline, and sutured each layer together.

\section{Assays of serum C3 level}

Mice were anaesthetized with pentobarbital intraperitoneally. Blood from each group was collected from mouse retroorbital sinus on days 3, 7, 14 and 28 post-implantation, separated to get serum. Serum C3 were analyzed using ELISA kits (Mlbio Systems, Shanghai, China). 


\section{Histological analysis}

Femoral condyles from the same mice as above section were removed on days 3 and 7 postimplantation, washed with saline, fixed in $4 \%$ neutral buffered formaldehyde for 4 days, decalcified with EDTA solution for 35 days, embedded in paraffin, sectioned at $8 \mu \mathrm{m}$ sections, stained with haematoxylin and eosin (H\&E) and TRAP (MilliporeSigma,Burlington, MA, USA), and observed using light microscopy (Olympus BX $51+$ DP71, Japan). The average grey scale of TRAP-positive was measured using a computer image analysis system image-pro plus 6.0.

\section{C3 gene expression assays by RT-qPCR}

Femoral condyles were removed on days $3,7,14$ and 28 post-implantation, extracted total RNA using TRIzol reagent (Takara, Otsu, Japan), and RT-qPCRs were performed using SYBRPremix Ex Taq $\otimes$ kit (TliRNaseH Plus) (Takara, Otsu, Japan) by ABI 7500 Real-Time PCR system (Thermo Fisher Scientific Inc, Foster city, CA, US). The primers used for RT-qPCR showed in Table 1. Data were normalized by $\beta$ actin expressions using the $2^{-\Delta \Delta C t}$ method.

\section{C3 protein expression assays by Western blot}

Femoral condyles were removed on days 3 and 7 post-implantation, extracted protein using Minute (TM) Total Protein kit (Invent, Eden Prairie, Minnesota, USA). Protein concentration was determined by BCA protein quantitative assay kit (Beyotime, Shanghai, China). $30 \mu \mathrm{g}$ of total protein was used to run $10 \%$ SDS-PAGE, then transferred to a polyvinylidene difluoride (PVDF) membrane. The membrane was blocked with $5 \%$ skimmed milk plus $0.1 \%$ tween 20 in $10 \%$ tris-buffered saline at room temperature for $2 \mathrm{~h}$, then incubated with primary anti C3 antibody (1:100, Abcam, Cambridge, MA, USA) or anti $\beta$-actin (1:2000, Abcam, Cambridge, MA, USA) at $4{ }^{\circ} \mathrm{C}$ for $18 \mathrm{~h}$, washed with $10 \%$ tris-buffered saline for three times, then incubated with secondary anti rabbit lgG horseradish peroxidase-conjugated antibody $s$ at room temperature for $1 \mathrm{~h}$ (1:5000, Zsbio, Beijing, China). Finally, blot was visualized using an ECL kit (Pierce, MA, USA) according to the manufacturer's instructions. The relative density of each blot was quantified using Image $J$ software (NIH, Bethesda, MD, USA).

\section{C3 Immunochemistry staining analysis of femoral condyle tissue}

Femoral condyles were removed on days 3 and 7 post-implantation, washed with saline, fixed in $4 \%$ neutral buffered formaldehyde for 4 days, decalcified with EDTA solution for 35 days. The explanted tissues were embedded in paraffin, sectioned at $8 \mu \mathrm{m}$ thick sections. Then, sections were incubated with primary anti $\mathrm{C} 3$ antibody $(1: 100)$ at $4{ }^{\circ} \mathrm{C}$ for $24 \mathrm{~h}$, washed with $1 \times$ PBS, were incubated with secondary antibody, washed with $1 \times$ PBS, stained with 3, 3-diaminobenzidin (DAB), and observed using light microscopy. The average grey scale of positive $\mathrm{C} 3$ was measured using an image analysis system imagepro plus 6.0.

\section{Activated complement pathway analysis}


Classical pathway maker $\mathrm{C} 1 \mathrm{q}$, alternative pathway marker $\mathrm{Bb}$ and mannose binding lectin marker MBL in the serum were analyzed using commercially available ELISA kits in accordance with the manufacturer's protocol (mlbio Systems, Shanghai, China).

\section{Cell culture}

Raw 264.7 cells, which is a macrophage cell line and differentiate into preosteoclasts and further osteoclasts in the osteoclast induction condition, were cultured with H-DMEM (Gibco, USA) containing $10 \%$ fetal bovine serum (FBS; Sigma, USA) at $37^{\circ} \mathrm{C}$ in a humidified $5 \% \mathrm{CO}_{2}$ atmosphere in general.

Osteoclast induction condition medium contained $30 \mathrm{ng} / \mathrm{mL}$ recombinant Murine Macrophage Colony Stimulating Factor (rmM-CSF) (R\&D system, Minneapolis, Minnesota,USA) and $50 \mathrm{ng} / \mathrm{mL}$ recombinant Murine Soluble Receptor Activator of NF-KB Ligand (rmRANKL) (R\&D system). Culture medium of complement group contained 10\% Guinea Pig serum (Pingrui, Beijing, China) because there are much rich complement proteins in the guinea pig serum.

Basically, all in vitro experiments were divided into three groups, complement group cultured with $\mathrm{H}$ DMEM plus osteoclast induction condition medium and $10 \%$ guinea pig serum (CM1), C3aR inhibitor group cultured with H-DMEM plus osteoclast induction condition medium and $10 \%$ guinea pig serum plus C3aR inhibitor (MCE, Monmouth Junction, New Jersey, USA) (CM2) and control group cultured with HDMEM plus osteoclast induction condition medium and $10 \%$ inactivated guinea pig serum (CM3).

\section{C3a receptor (C3aR) and C3b receptor (CR1) gene expressions in osteoclasts in vitro}

Raw 264.7 cells were cultured with H-DMEM (Gibco, USA) containing $10 \%$ fetal bovine serum, $30 \mathrm{ng} / \mathrm{mL}$ rmM-CSF and $50 \mathrm{ng} / \mathrm{mL}$ rmRANKL for 4 days using as osteoclasts. Then, profiles of C3aR and CR1 gene expressions in osteoclasts were measured by RT-qPCR.

\section{Osteoclast formation assay in vitro}

Raw 264.7 cells were cultured at $1 \times 10^{4}$ cells/well in 24-well plate for $24 \mathrm{~h}$ which there was a cell climbing slide in each well. Culture medium was replaced with $\mathrm{CM} 1, \mathrm{CM} 2$ or $\mathrm{CM} 3$ respectively, and cultured for 5 days, then stained with TRAP.

\section{Evaluating C3a secretion from osteoclasts after Ti implantation in vitro}

The 6-well plates were pretreated with 1\% BSA blocking solution for $60 \mathrm{~min}$. Afterward, $10 \mathrm{mM}$ ethylenediaminetetraacetic acid (EDTA) was added to stop any further complement activation. This experiment was divided into three groups as above cell culture section. Cells were cultured for 3 days. Then, supernatants from each group was analyzed for C3a using ELISA kit (Boster, Wuhan, China).

\section{Evaluating pre-inflammatory cytokine secretion from osteoclasts in vitro}


This experiment was divided into six groups, group one was Raw264.7 with titanium+CM1, group two was Raw264.7 with titanium+CM2, group three was Raw264.7 with titanium+CM3, group four was Raw264.7+CM1, group five was Raw264.7+CM2 and group six was Raw264.7+CM3. TNF-aand MMP9 in the supernatant from each group were analyzed using ELISA kits (Boster, Wuhan, China).

\section{Gene expression profiles of Osteoclast related genes by RT-qPCRand Western blot assays}

Cells from each group of above section were used to perform RT-qPCR assays. Primers used for RT-qPCR were showed in Table 1.

Table 1 Primers used in this study

Gene Forward primer (5'-3') Reverse primer (5'-3')
C3 CCGTGAACAGGAGGAACTTAAGG ATGCTGCAGAAGGCTGGATT
C3ar TCACCCTGGCCGATTTCCT GATGGATGGGATAAGTTTGCACA
CR1 AACACATGGTTACCAGGTGTACC CGTGCCTCTCCAGCCATAAG
NF-KB1 CCGGGATCCGCAAACTCAGCTTTAC CGGAATTCGTGGCGACCGTGATACC
NFATC1 CAAGTCTCACCACAGGGCTCACTA TCAGCCGTCCCAATGAACAG
B actin AGGAGCAATGATCTTGATCTT TGCCAACACAGTGCTGTCT

Total protein was extracted by the RIPA cell lysate (Solarbio, Beijing, China). Western blots were performed as previous section. Primary antibodies, anti NF-KB (1:5000, Abcam, Cambridge, MA, USA) and anti NFATc1 (1:5000, Abcam, Cambridge, MA, USA) were used.

\section{Statistical analysis}

The values were expressed as the mean \pm standard deviation (SD), and Student's t-tests were used to discern significant differences among the groups using the SPSS 21.0 software. A probability value (p) of less than 0.05 was considered statistically significant.

\section{Results}

\subsection{Effects of Ti implantation on inflammatory responses and osteoclasts}

One reason caused the failure of $\mathrm{Ti}$ implantation is the local inflammatory responses ${ }^{[13]}$. Therefore, first we evaluated if $\mathrm{Ti}$ implant could stimulate the local inflammatory responses around the bone tissue in our model system. Indeed, H\&E staining revealed that there were more blue inflammatory cells in the 
surrounding tissues of both Ti implant and defect areas on days 3 and day7 compared to control group (Figure $1 \mathrm{~A}$ ). Further, data from TRAP staining also showed that TRAP-positive osteoclasts significantly increased in the surrounding tissue of the Ti implant and defect area on days 3 and day 7 compared to control group ( $P \otimes 0.05$ ) (Figures 1B and 1C). These results suggest that Ti implant triggers local inflammation leading to osteoclast activation in the local tissue at the early phase of implantation.

\subsection{Effects of Ti implantation on C3 in serum and tissue}

It is known that a critical first step of C3 mediated recognition and opsonization to foreign biomaterials is the subsequent inflammatory response ${ }^{[14]}$. Interestingly, above H\&E staining showed that the Ti implant could cause local inflammatory responses. Therefore, next it was important to know if the Ti implant could influence C3 expression. ELISA data demonstrated that C3 protein was significantly increased in the serum of $\mathrm{Ti}$ implant treated mice and defect mice, especially $\mathrm{Ti}$ implant treated mice which were significant higher than that of defect mice compared to control mice till day 14 post-implantation and the peak of C3 protein in the serum was day 7 (Figure 2A) (Pख0.05). RT-qPCR analysis further showed that C3 expression was significantly higher in the surrounding tissue of Ti implant and defect area compared to control mice till day 7 ( $P \otimes 0.05)$ while there was no difference between groups after 14 days (Figure 2B). Next, Western blot assay also confirmed that the C3 protein level was clearly higher than that of control group in the surrounding tissue of Ti implant and defect area on days 3 and 7 (Figures 2C and 2D). Finally, data of immunohistochemical staining further demonstrated that C3 expression was indeed higher compared to control mice in the surrounding tissue of Ti implant and defect area on days 3 and 7 (Figures 2E and 2F). These data suggest that Ti implant and defect model can increase $\mathrm{C} 3$ expression systemically and locally, especially Ti implant. Interestingly, C3 expression consistent with results of inflammatory responses and osteoclast level.

\subsection{Effects of higher C3 expression on complement pathways post Ti implantation}

Complement $\mathrm{C} 1 \mathrm{q}$ is a protein complex together with $\mathrm{C} 1 \mathrm{r}$ and $\mathrm{C} 1 \mathrm{~s}$ which activation can initiate the classical complement pathway ${ }^{[15]}$. Complement factor $B$ is the component of the alternative pathway of complement activation ${ }^{[15]}$. The active subunit $\mathrm{Bb}$ associates with $\mathrm{C} 3 \mathrm{~b}$ to form convertase of the

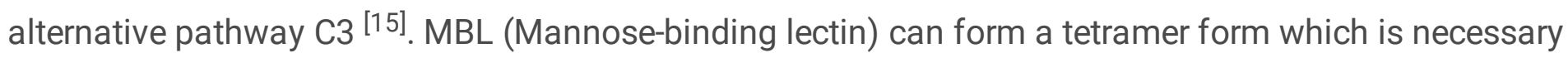
for activation of complement ${ }^{[15]}$. Therefore, to understand the role of the complement activation pathway in the $\mathrm{C} 3$ activation after $\mathrm{Ti}$ implantation, $\mathrm{C} 1 \mathrm{q}, \mathrm{Bb}$ and $\mathrm{MBL}$ levels in the serum were evaluated by ELISA (Figure 3). Data showed that the C1q was significantly increased compared to control mice only on day 3 post Ti implantation (Figure 3A) (Pख0.05), and Bb was significantly increased compared to control mice on days 3,7 and 14 post Ti implantation (Figure 3B) (P凶0.05) while there was no difference of MBL level in the serum at different time points (Figure $3 \mathrm{C}$ ). These results clearly suggest that alternative pathway and classical pathways at very early time point (day 3), rather than MBL pathway, play predominate roles in the activation of complement system after post Ti implantation, and only alternative pathway plays predominate roles in the activation of complement system after days 7 and 14 post Ti implantation. 


\subsection{Expression profiles of C3aR and CR1 effects of C3a/C3b on osteoclasts}

Osteogenesis can be improved by $\mathrm{C} 3$ fragment ${ }^{[12]}$. Figure 4A demonstrates that the C3aR (C3a receptor) gene expression was dramatically increased compared to CR1 (complement receptor type 1, C3b receptor) expression in the osteoclast ( $P \otimes 0.05)$. Raw 264.7 cell cultured with osteoclast medium plus guinea pig serum (complement group) showed extensively aggregated osteoclasts with ameboid morphology (Figure 4B) compared to control group (Figure 4D). The C3aR inhibitor, however, could block the osteoclast cluster formation (Figure 4C). These data indicate that complements, especially C3a can promote osteoclasts to aggregate and form osteoclast cluster.

\subsection{Effects of C3a on inflammatory cytokines of osteoclasts}

In vivo animal experiments demonstrated that the Ti implant caused inflammation through C3 complement systemically and locally (Figures 1,2 and 3). Further in vitro experiments showed that the C3a was the important one from C3 (Figure 4). To further understand which cytokines were influenced, in vitro osteoclast model was used to evaluate changes of TNF-a and MMP-9. TNF- $a$ is an inflammatory cytokine that is released by a variety of cell types, including macrophages, lymphocytes, and neutrophils. Levels of TNF-a are increased in many inflammatory diseases ${ }^{[16]}$. MMP-9 is also an important cytokine to regulate inflammatory responses ${ }^{[17]}$. First, data showed that the Ti implant promoted significant secretion of $\mathrm{C3}$ a into supernatant in the osteoclast culture system (Figure 5A). Data from cytokine assays demonstrated that the Ti implant could significantly cause TNF-a and MMP-9 increases from osteoclasts, and the increases were mediated by the C3a compared to different control groups (Figures 5B and 5C). These results indicate that the $\mathrm{C} 3 \mathrm{a}$ can be activated by the $\mathrm{Ti}$ implant resulting in the increases of certain cytokine secretions.

\subsection{Reletionship between C3a and transcription factors, NF-KB and NFATc1 in the co-culture with Ti implant in vitro}

It is known that the transcription factors, NF-KB and NFATc1 are involved in cytokine regulation ${ }^{[18]}$. Therefore, next we evaluated if NF-KB and NFATc1 were influenced by Ti implant and C3a. Data of RTqPCR assays showed that the C3a significantly increased expressions of NF-KB and NFATc1 compared to control groups (Figures $6 \mathrm{~A}$ and $6 \mathrm{~B}$ ) while the $\mathrm{C} 3 \mathrm{a}$ only was not enough to increase expressions of NF-KB and NFATc1 at the protein levels (Figures 6C, 6D and 6E). Interestingly, RT-qPCR assays demonstrated that the C3a plus Ti implant dramatically increased expressions of NF-KB and NFATc1 compared to control groups (Figures 6A and 6B) and also increased expressions of NF-KB and NFATc1 at the protein levels (Figures $6 \mathrm{C}, 6 \mathrm{D}$ and $6 \mathrm{E}$ ). These results indicate that the $\mathrm{C} 3 \mathrm{a}$ only can increase inflammatory cytokines mildly, and the C3a plus Ti implant will strongly enhance productions of inflammatory cytokines via increases of transcription factors NK-kb and NFATc1.

\section{Discuss}


Ti implantation is a very useful dental replacement despite the bone resorption due to inflammatory responses. Complement system plays important roles in the development and homeostasis of healthy bone ${ }^{[7]}$. Complement $\mathrm{C} 3$ is a convergence of three distinct complement activation pathways, and an orchestrator for local inflammatory responses via the initiation of both innate immunity and adaptive immunity ${ }^{[19]}$. In this study, we evaluated if the complements, especially C3 involved in the inflammatory responses after Ti implantation. Our data demonstrate that the C3a indeed play important roles in the inflammatory responses through transcription factors NF-kB and NFATc1, especially with Ti implant together resulting in the osteoclast differentiation and proliferation, which most likely mechanism causes the failure of Ti implantation.

The C3 plays important roles in the pathogenesis of chronic inflammatory diseases by affecting bone remodeling of the host. It has been shown that deposits of C3 in gingival crevicular fluid can modulate periodontitis disease development and progression ${ }^{[20]}$, furthermore, deposits of C3 are also found in the synovial vasculature and the intercellular matrix in both rheumatoid arthritis (RA) and acute arthritis ${ }^{\text {[21] }}$. Our results are consistent with these studies. The $\mathrm{C} 3$ level was higher in the serum and local tissues after $\mathrm{Ti}$ implantation (Figure 2). In general, $\mathrm{C} 3$ can be produced by hepatocytes and macrophage in response to inflammatory stimulation. In addition, the activated osteoclasts can produce $\mathrm{C} 3$ fragment that stimulates osteoblast differentiation ${ }^{[10]}$. We also find that the osteoclasts in our co-culture cell model with $\mathrm{Ti}$ implant can produce the $\mathrm{C} 3 \mathrm{a}$ (Figure 5).

C3 serves as convergence of three pathways of complement system. Previous report demonstrated that $\mathrm{Ti}$ implantation into the bone of oral cavity could trigger C3 activation predominantly via both classical and alternative pathways ${ }^{[22]}$. Our results also suggest clearly that alternative pathway and classical pathway play predominate roles after days 3 post $\mathrm{Ti}$ implantation, and only alternative pathway plays predominate roles in the activation of complement system after days 7 and 14 post Ti implantation. These data suggest that the complement activation cascade of classical and alternative pathways are responsible for the $\mathrm{C} 3$ induced inflammatory responses post $\mathrm{Ti}$ implantation.

Abnormal osteoclast activation can cause the bone resorption leading to the osteoporosis in RA ${ }^{[23]}$. The C3 is required for the proliferation of precursor cells and early differentiation of osteoclast, and promotes osteoclast formation. In contrast, blockade of C3 by anti-C3 antibody remarkably inhibited osteoclastogenesis in vitro [24]. Interestingly, our data also demonstrate that Ti implantation up-regulates the $\mathrm{C} 3$ resulting in inflammatory reaction and osteoclast formation (Figures 1 and 2).

C3-convertase cleaves and activates component C3, generating C3a and C3b. Both of C3a and C3b mediate inflammatory responses of the local tissue after biomaterial implantation ${ }^{[14,25]}$ Transcriptional factors, NF-kB and NFAT are responsible for the inflammatory cytokine expression in the osteoclast of bone tissue ${ }^{[26]}$. Interestingly, our data show that the C3a, but no C3b is actively and predominantly involved in the osteoclast formation in vitro. Increase of the $\mathrm{C} 3 \mathrm{a} / \mathrm{C} 3 \mathrm{aR}$ from the osteoclasts by Ti implant stimulates secretions of pro-inflammatory cytokines TNF-a and MMP9 in vitro (Figures 4 and 5). Also, we 
found out that the increases of pro-inflammatory cytokines, TNF-a and MMP9 are mediate by the increases of transcriptional factors, NF-kb and NFATs (Figure 6). In fact, it has been shown that titanium stimulates the generation of both $\mathrm{C} 3 \mathrm{a}$ and $\mathrm{C} 3 \mathrm{~b}$, which are cleaved from C3 in human serum and plasma [27]. Therefore, these results suggest that the modification of Ti implant may control the magnitude of complement activation in order to achieve the stability of Ti implant in vivo.

\section{Conclusion}

Our study demonstrates that the $\mathrm{Ti}$ implant stimulates the $\mathrm{C} 3 / \mathrm{C} 3 \mathrm{a}$ expressions in vivo and in vitro, which are further induce pro-inflammatory cytokines of TNF- $a$ and MMP9 via transcription factors NF-kB and NFATc1 resulting in the differentiation and proliferation of osteoclasts in vivo and in vitro, bone resorption of surrounding tissues of Ti implant and the failure of Ti implantation. Therefore, our study proposes a novel method, blocking C3a activation to prevent bone resorption and prolong the survival of Ti implant.

\section{Declarations}

\section{Acknowledgments}

This study was supported by grants from the National Key R\&D Program of China 2016 YFC1102800, the National Natural Science Foundation of China (NSFC) (No.81870741).

\section{Conflicts of Interest}

All authors have no conflicts of interest to declare.

\section{References}

[1] Liu XH, Wu L, Ai HJ, Han Y, Hu Y. Cytocompatibility and early osseointegration of nanoTiO 2 -modified Ti-24 Nb-4 Zr-7.9 Sn surfaces. Mater Sci Eng C, 2015(48):256-262.

[2] Tresguerres FGF, Torres J, López-Quiles J, Hernández G, Vega JA, Tresguerres IF. The osteocyte: A multifunctional cell within the bone. Ann Anat, 2020, 227: 151422

[3] Giang J, Seelen MAJ, van Doorn MBA, Rissmann R, Prens EP, Damman J. Complement Activation in Inflammatory Skin Diseases. Front Immunol, 2018, 9: 639.

[4] Holers VM, Banda NK. Complement in the initiation and evolution of rheumatoid arthritis. Front Immunol, 2018, 9: 1057.

[5] Vignesh P, Rawat A, Sharma M, Singh S. Complement in autoimmune diseases. Clin Chim Acta, 2017, 465: 123-130. 
[6] López-Lera A, Corvillo F, Nozal P, Regueiro JR, Sánchez-Corral P, López-Trascasa M. Complement as a diagnostic tool in immunopathology. Semin Cell Dev Biol, 2019, 85: 86-97.

[7] Mödinger Y, Löffler B, Huber-Lang M, Ignatius A. Complement involvement in bone homeostasis and bone disorders. Semin Immunol, 2018, 37: 53-65.

[8] Noris M, Remuzzi G. Overview of complement activation and regulation. Seminars in Nephrology, 2013, 33 (6): 479-492.

[9] Wu F, Zou Q, Ding X, Shi D, Zhu X, Hu W, Liu L, Zhou H. Complement component C3a plays a critical role in endothelial activation and leukocyte recruitment into the brain. J Neuroinflammation, 2016, 13:23.

[10] Matsuoka K, Park KA, Ito M, Ikeda K, Takeshita S. Osteoclast-derived Complement Component 3a Stimulates Osteoblast Differentiation. J Bone Miner Res, 2014, 29(7):1522-30.

[11] Ignatius A, Schoengraf P, Kreja L, Liedert A, Recknagel S, Kandert S, Brenner RE, Schneider M, Lambris JD, Huber-Lang M. Complement C3a and C5a modulate osteoclast formation and inflammatory response of osteoblasts in synergism with IL-1 $\beta$. J Cell Biochem, 2011, 112(9): 2594-2605.

[12] Ehrnthaller C, Huber-Lang M, Nilsson P, Bindl R, Redeker S, Recknagel S, Rapp A, Mollnes T, Amling M, Gebhard F, Ignatius A. Complement C3 and C5 deficiency affects fracture healing. PLoS One, 2013, 8(11): e81341.

[13] Eger M, Hiram-Bab S, Liron T, Sterer N, Carmi Y, Kohavi D, Gabet Y. Mechanism and Prevention of Titanium Particle-Induced Inflammation and Osteolysis. Front Immunol, 2018,9:2963.

[14] McLaren JS, Macri-Pellizzeri L, Hossain KMZ, Patel U, Grant DM, Scammell BE, Ahmed I, Sottile V. Porous phosphate-based glass microspheres show biocompatibility, tissue infiltration, and osteogenic onset in an ovine bone defect model. ACS Appl Mater Interfaces, 2019,11(17):15436-15446.

[15] Dobó J, Kocsis A, Gál P. Be on target: strategies of targeting alternative and lectin pathway components in complement-mediated diseases. Front Immunol, 2018,9:1851.

[16] Deora A, Hegde S, Lee J, Choi CH, Chang Q, Lee C, Eaton L, Tang H, Wang D, Lee D, Michalak M, Tomlinson M, Tao Q, Gaur N, Harvey B, McLoughlin S, Labkovsky B, Ghayur T. Transmembrane TNFdependent uptake of anti-TNF antibodies. MAbs, 2017,9(4):680-695.

[17] Zhu SH, Liu BQ, Hao MJ, Fan YX, Qian C, Teng P, Zhou XW, Hu L, Liu WT, Yuan ZL, Li QP. Paeoniflorin suppressed high glucose-induced retinal microglia MMP-9 expression and inflammatory response via inhibition of TLR4/NF-KB pathway through upregulation of SOCS3 in diabetic retinopathy. Inflammation, 2017,40(5):1475-1486.

[18] Zhang Y, Xu S, Li K, Tan K, Liang K, Wang J, Shen J, Zou W, Hu L, Cai D, Ding C, Li M, Xiao G, Liu B, Liu A, Bai X. mTORC1 inhibits NF-KB/NFATc1 signaling and prevents osteoclast precursor differentiation, in 
Vitro and in Mice. J Bone Miner Res, 2017,32(9):1829-1840.

[19] Wenzel UO, Bode M, Köhl J, Ehmke H. A pathogenic role of complement in arterial hypertension and hypertensive end organ damage. Am J Physiol Heart Circ Physiol, 2017,312(3):H349-H354

[20] Hughes S, Gumas J, Lee R, Rumano M, Berger N, Gautam AK, Sfyroera G, Chan AL, Gnanaguru G, Connor KM, Kim BJ, Dunaief JL, Ricklin D, Hajishengallis G, Yancopoulou D, Reis ES, Mastellos DC, Lambris JD. Prolonged intraocular residence and retinal tissue distribution of a fourth-generation compstatin-based C3 inhibitor in non-human primates. Clin Immunol, 2020, 214:108391.

[21] Romano C, Del Mastro A, Sellitto A, Solaro E, Esposito S, Cuomo G. Tocilizumab reduces complement $\mathrm{C} 3$ and $\mathrm{C} 4$ serum levels in rheumatoid arthritis patients.

Clin Rheumatol, 2018, 37(6):1695-1700.

[22] Mödinger Y, Teixeira GQ, Neidlinger-Wilke C, Ignatius A. Role of the Complement System in the Response to Orthopedic Biomaterials. Int J Mol Sci, 2018, 19(11):3367.

[23] Holers VM, Banda NK. Complement in the initiation and evolution of rheumatoid arthritis. Front Immunol, 2018, 9: 1057.

[24] Tu Z, Bu H, Dennis JE, Lin F. Efficient osteoclast differentiation requires local complement activation. Blood, 2010, 116(22):4456-4463.

[25] loannis K, Stavros R, Robert A. Inhibition of biomaterial-induced complement activation attenuates the inflammatory host response to implantation. FASEB J, 2013, 27(7): 2768-2776.

[26] Su YW, Chim SM, Zhou L, Hassanshahi M, Chung R, Fan C, Song Y, Foster BK, Prestidge CA, Peymanfar Y, Tang Q, Butler LM, Gronthos S, Chen D, Xie Y, Chen L, Zhou XF, Xu J, Xian CJ. Osteoblast derived-neurotrophin-3 induces cartilage removal proteases and osteoclast-mediated function at injured growth plate in rats. Bone, 2018, 116:232-247.

[27] Arvidsson S, Askendal A, Tengvall P. Blood plasma contact activation on silicon, titanium and aluminium. Biomaterials, 2007, 28(7):1346-1354.

\section{Figures}



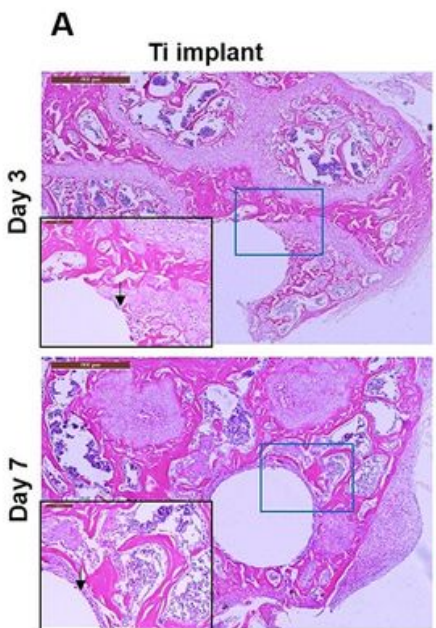

B
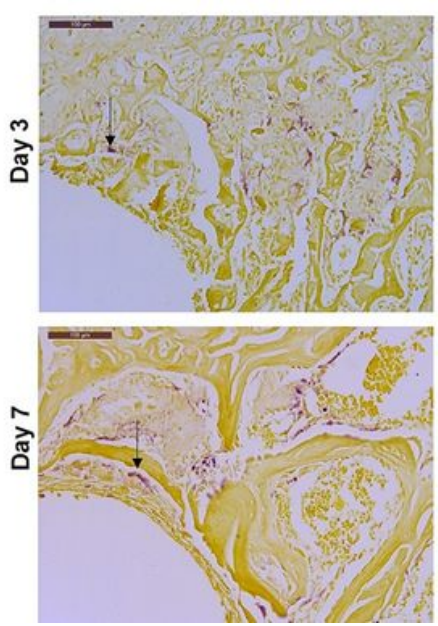

Defect
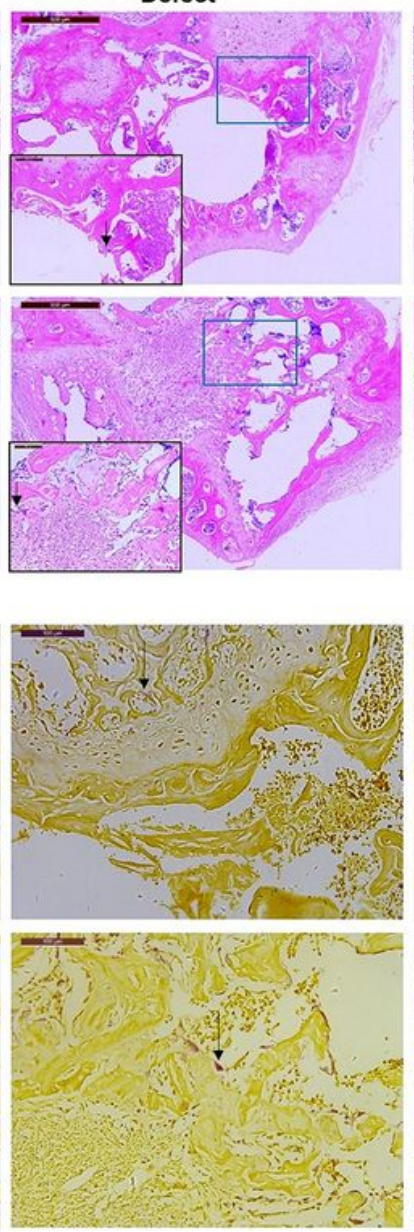

Control
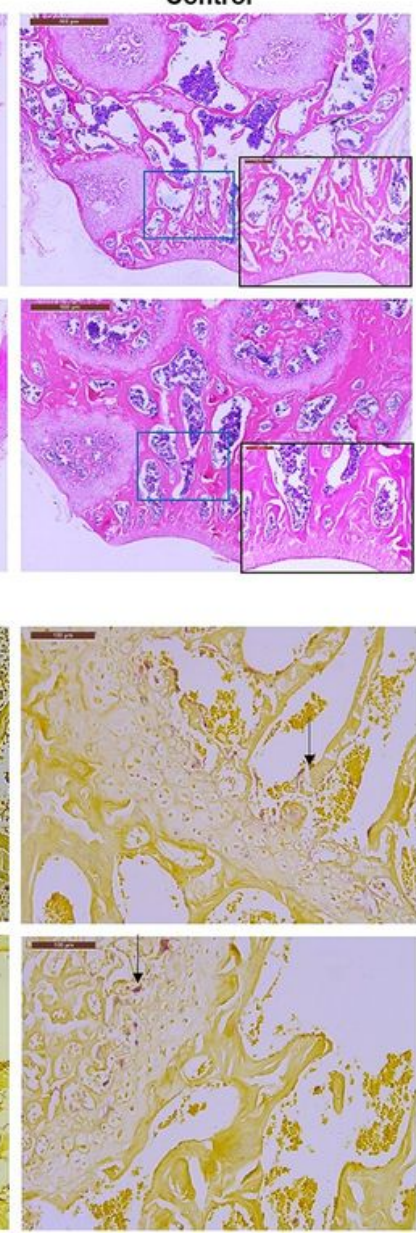

C

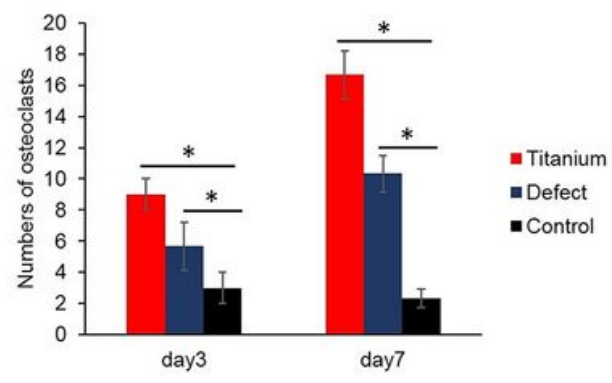

Figure 1

Inflammatory responses and Osteoclast activation after titanium implantation A. H\&E staining of femoral condyle after 3 and 7 days post-implantation. B. TRAP staining of femoral condyle after 3 and 7 days post-implantation. C. Bar graph of osteoclast numbers for TRAP staining. 
A

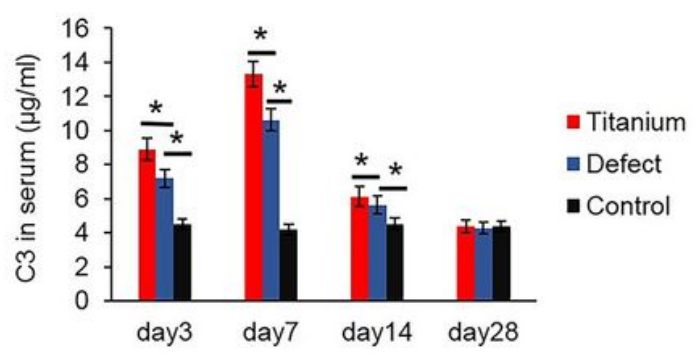

C

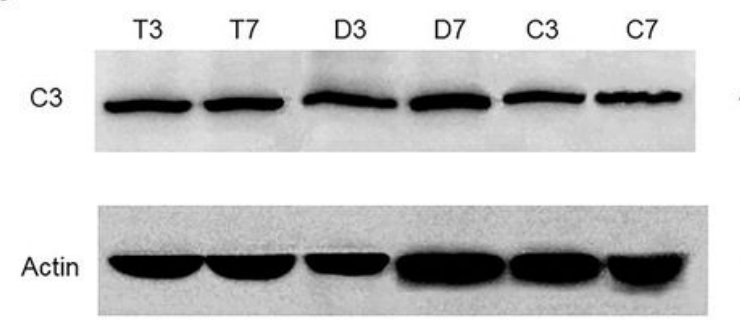

43KD

E
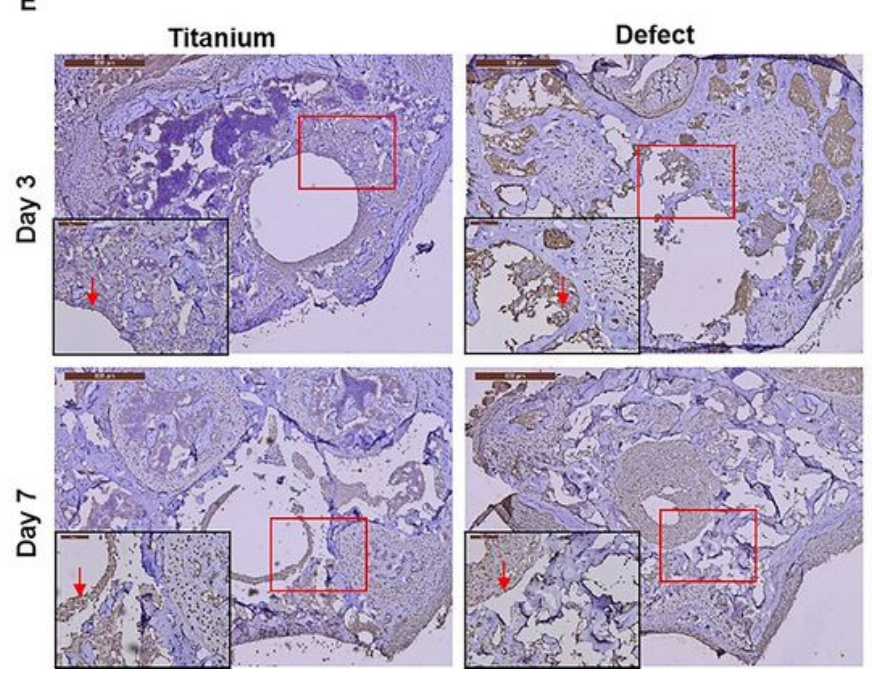

B

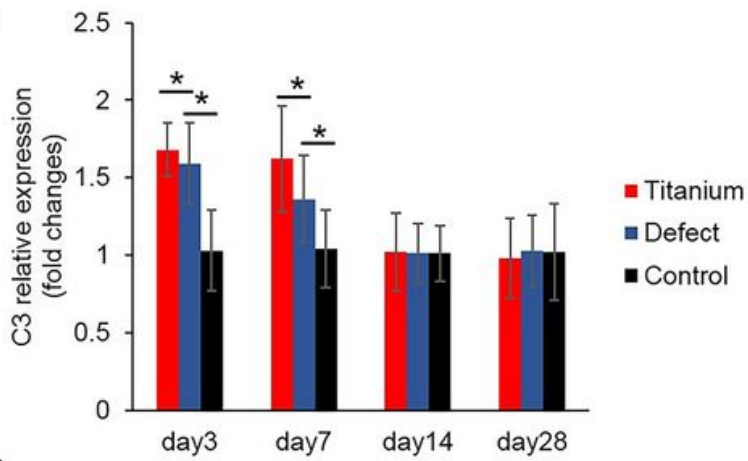

D

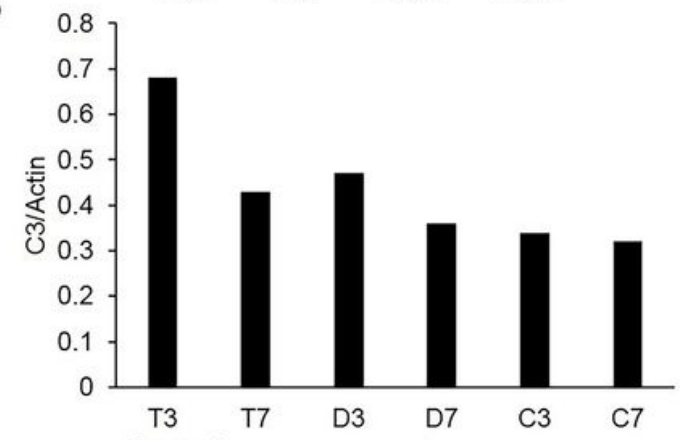

\section{Figure 2}

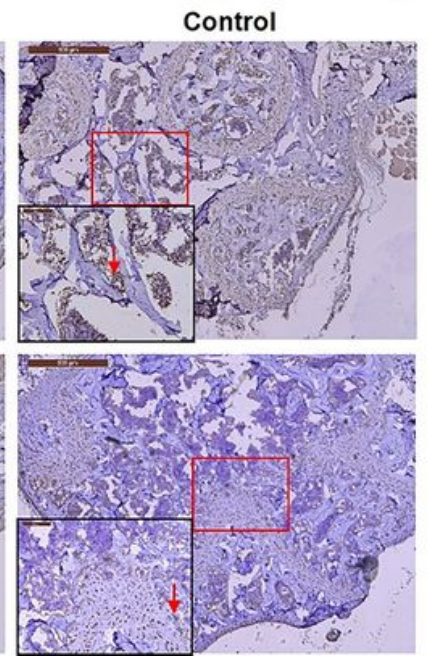

$F$

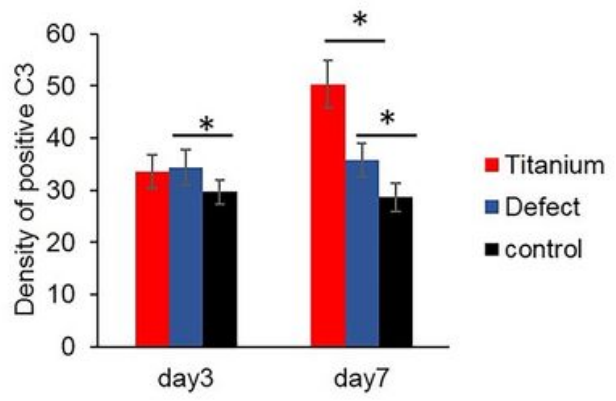

Profile of C3 expression in serum and femoral condyle tissue. A. C3 protein level in the serum after post implantation. B. C3 gene expression in the femoral condyle tissue after post implantation. C. Western blot of C3 protein from femoral condyle tissue after post implantation. D. Bar graph of quantitative Western blot. E. C3 immumohistochemical staining of femoral condyle tissue. F. Bar graph of quantitative C3 immumohistochemical staining. D3, 3 day defect group, D7, 7 day defect group, T3, 3 day Ti group, T7, 7 day Ti group, C3, 3 day control group, C7, 7 day control group. *, P『0.05. 

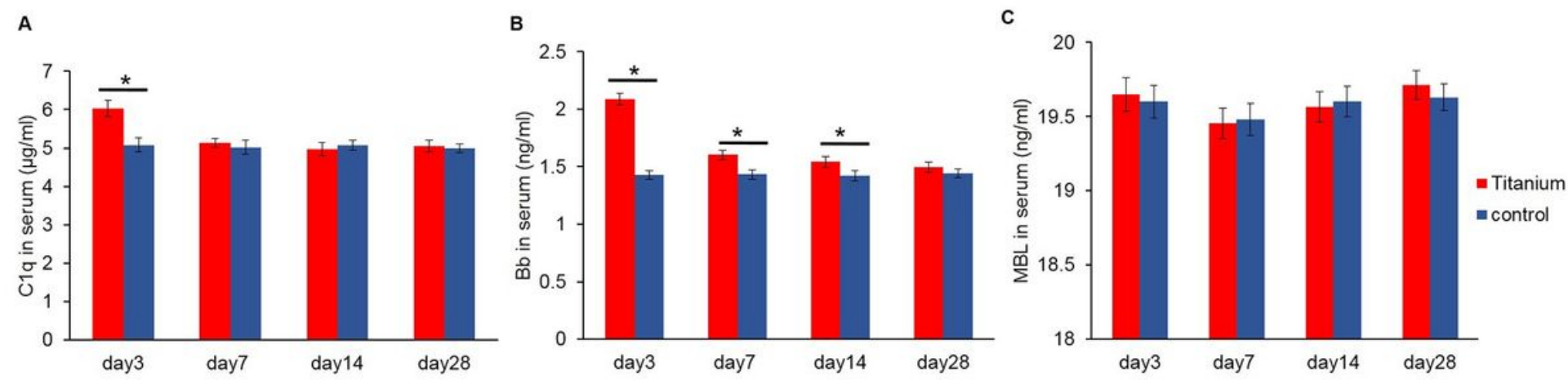

Figure 3

Possible complement pathways involved in $\mathrm{C} 3$ activation. A. $\mathrm{C} 1 \mathrm{q}$ level in the serum. $\mathrm{B}$. Bb level in the serum. C. MBL level in the serum. *, Pख0.05.
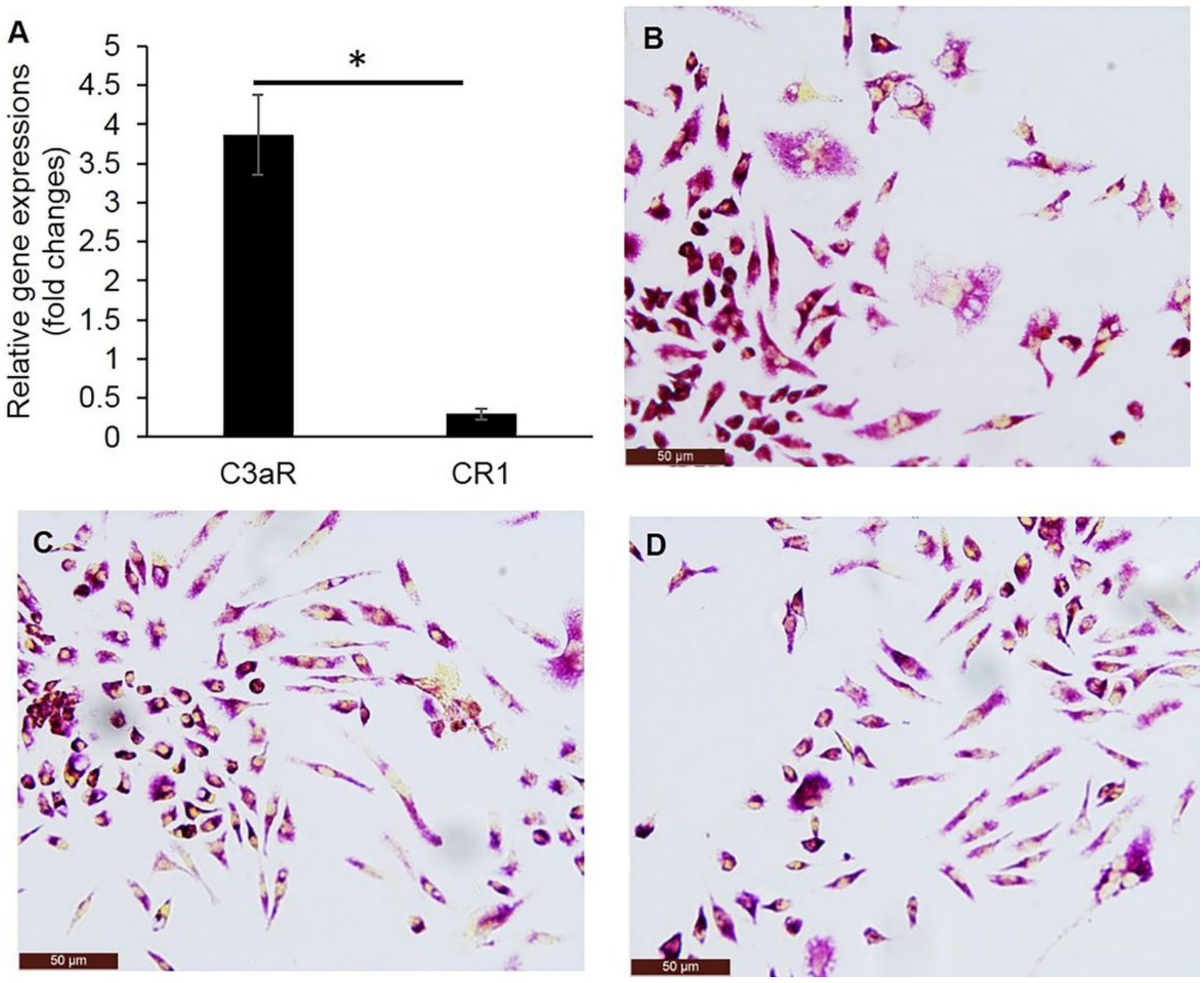

Figure 4 
Difference effect on C3aR and CR1 of osteoclasts. A. C3aR and CR1 gene expressions in osteoclasts after 5 days. B. TRAP staining of complement group. C. TRAP staining of C3aR inhibitor group. D. TRAP staining of control group. *, $\mathrm{P} \otimes 0.05$
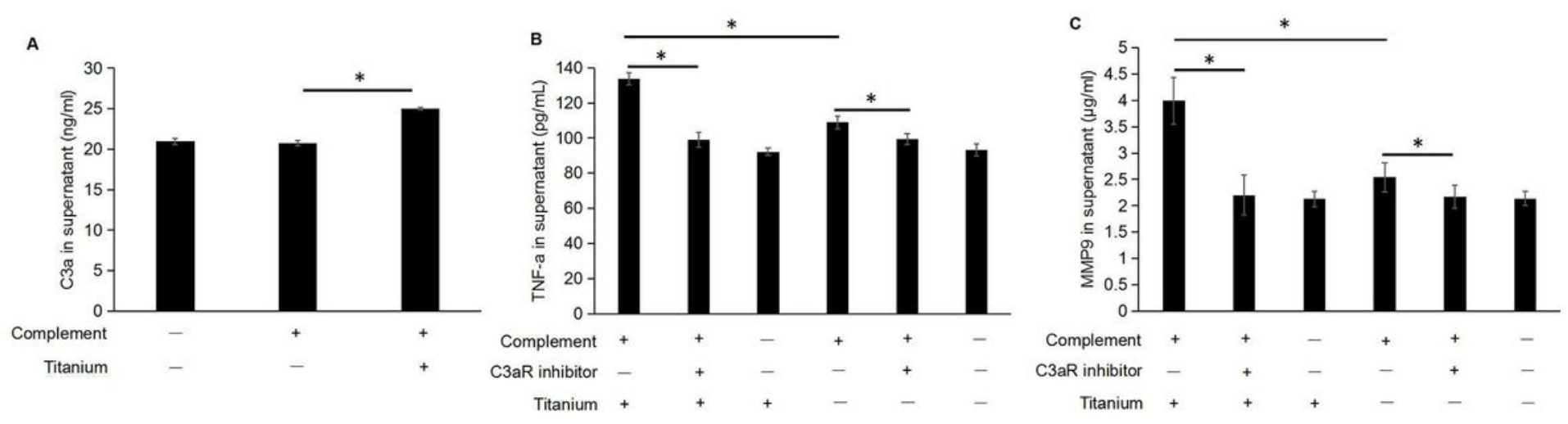

Figure 5

Evaluation of C3a secretion and C3a effects on cytokines during Ti implant co-culture with osteoclasts. A. C3a level in the supernatant by ELISA. B. TNF-a level in the supernatant by ELISA. C. MMP-9 level in the supernatant by ELISA. *, Pख0.05

A

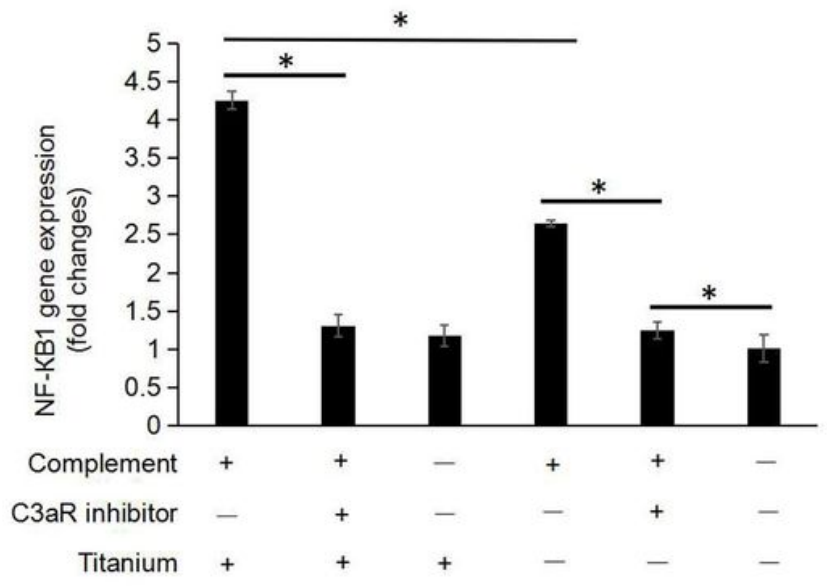

C

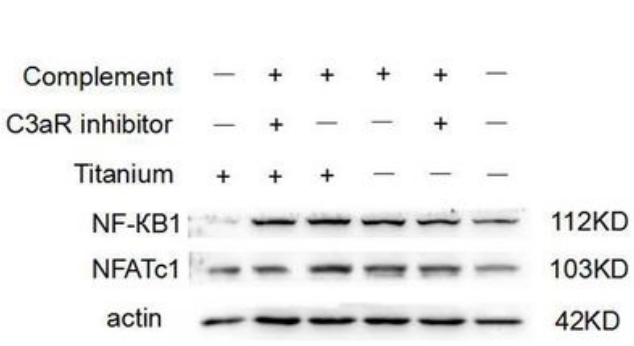

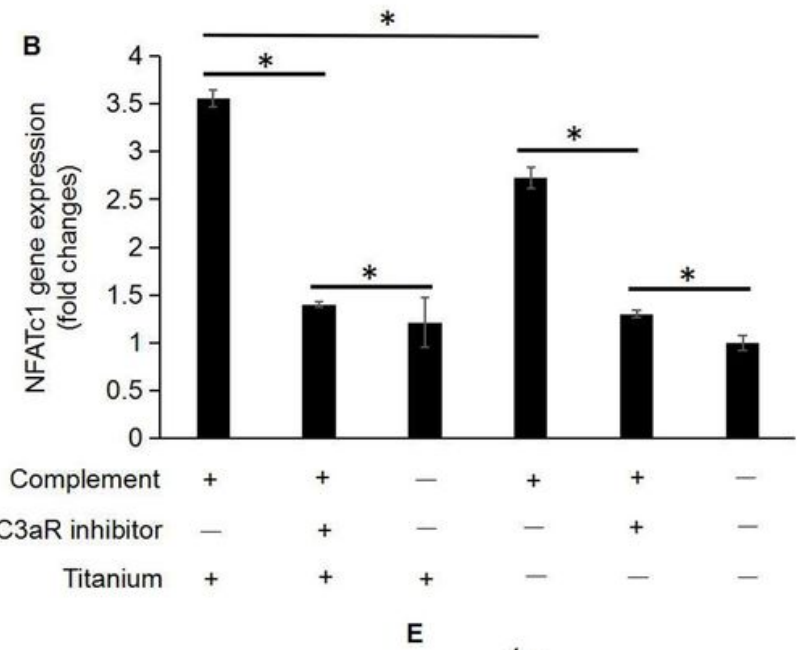

D

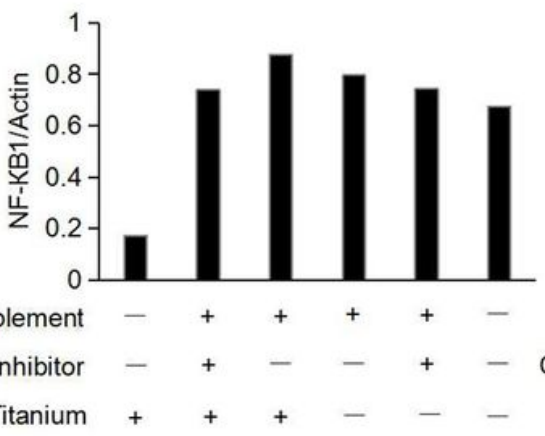

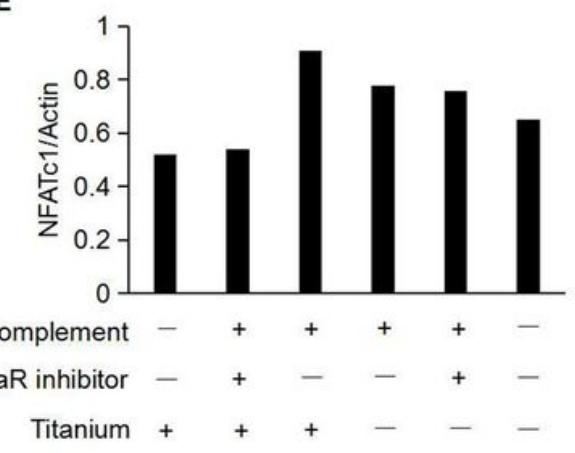

Figure 6

Profiles of NF-KB1 and NFATc1 expressions after Ti implant co-culture. A. RT-qPCR data of NF-KB1. B. RTqPCR data of NFATc1. C. Western blot analyses of NF-KB1 and NFATc1 expressions. D. Bar graph of 
quantitative analysis of NF-KB1 Western blot. D. Bar graph of quantitative analysis of NFATc1 Western blot. *, Pख0.05 